\title{
ANÁLISE DE PARÂMETROS BIOQUÍMICOS DO SANGUE DE CAMUNDONGOS TRATADOS COM LIDOCAÍNA
}

\author{
Biochemical parameters analysis of mice blood \\ treatred with lidocaine
}

\author{
Ana Paula Avelar Gallieri ${ }^{1}$ \\ André Wasicky² \\ Vergimari Rodrigues ${ }^{3}$ \\ Selen e Elifio-Esposito ${ }^{4}$ \\ Luiz Fernando Pereira ${ }^{5}$
}

\section{Resumo}

Estudos anteriores sobre os efeitos colaterais da Lidocaína mostraram que ao ser administrada cronicamente em camundongos, causava lesões nos rins e no fígado. Neste trabalho, avaliou-se, por meio de testes bioquímicos (Bilimubina, Transaminase Pirúvica -TGP, Transaminase Oxalacética - TGO e Creatinina), a hepatotoxicidade e a nefrotoxicidade do anestésico. Em nossas condições experimentais e, após administrações diárias durante 14 dias, não se observaram alterações significativas em tais marcadores bioquímicos plasmáticos.

Palavras-chave: Lidocaína; Bilirrubina; Transaminase pirúvica (ALT); Transaminase oxalacética (AST); Creatinina.

\footnotetext{
Abstract

Laboratório de Fisiologia Animal; Pontifícia Universidade Católica do Paraná.

Bióloga - PUCPR, ana_gallieri@hotmail.com.

2 Farmacêutico-Bioquímico- PUCPR, andrewasicky@yahoo.com.

3 Farmacêutica-Bioquímica - PUCPR, Vergimari.Rodrigues@gelita.com.

4 Prof.- de Fisiologia Animal - PUCPR, selene.e@pucpr.br.

5 Prof. de Fisiologia - PUCPR, fernando.pereira@pucpr.br.
}

Previous studies about Lidocaine side effects on mice showed, hystopathological injuries on kidney and liver, when the drug was administered as chronic treatment. In this work four biochemical parameters of hepatotoxicity and nephrotoxicity were analysed: bilirrubin, pyruvic transaminase, oxalacetic transaminase and creatinine. In our experimental conditions the results showed that after 14 days of daily administrations of Lidocaine did not cause significant alteration in such biochemicals parameters.

Keywords: Lidocaine; Bilirrubin; Pyruvic transaminase; Oxalacetic transaminase; Creatinine. 


\section{Introdução}

Anestésicos locais são comumente utilizados pelos cirurgiões-dentistas para procedimentos invasivos ou não. Os principais são a Lidocaína e a Prilocaína, pertencentes ao grupo amida $(1,2)$. São substâncias que, em contato com uma fibra nervosa, bloqueiam reversivelmente os canais de sódio, alterando os potenciais de membrana. As sensações que vêm da periferia para o sistema nervoso central (aferentes) e as mensagens que 0 sistema nervoso central envia para a periferia (eferentes) deixam de ser conduzidas pelo nervo sob ação do anestésico local, diminuindo as diversas formas de sensibilidade (tátil, térmica, dolorosa) além da atividade motora $(3,4)$. A ação dos anestésicos locais depende do estado de saúde do paciente, a qual pode interferir na farmacocinética e na farmacodinâmica desses agentes, aumentando assim o risco de toxicidade $(5,6)$. As reações adversas dos anestésicos locais são proporcionais à sua concentração na corrente sangüínea e dependem de sua natureza, da dose administrada, do local da administração e da técnica empregada $(7,8)$. Um ponto a ser salientado é o de que os anestésicos locais não causam lesões renais em pacientes sadios, porém, podem potencializar lesões renais preexistentes (9).

A Lidocaína, sintetizada por Löfgren, em 1943, resultou de pesquisas, cujo objetivo era reduzir a irritação local e os danos teciduais produzidos pelos anestésicos locais de uso corrente na época. Classificada como agente de potência e duração intermediária, é considerada protótipo para o estudo da potência e toxicidade de novos agentes anestésicos que vêm sendo sintetizados (1014). Também é empregada no tratamento de arritmias cardíacas de origem ventricular. Sabe-se que, em virtude de seu efeito estabilizador sobre as membranas excitáveis, diminui a velocidade de condução e a força de contração do miocárdio (15). Um dos pontos que sempre mereceu atenção especial é o potencial tóxico dessas drogas sobre 0 fígado e os rins $(16,17,18)$. A literatura aborda efeitos nefrotóxicos e hepatotóxicos da Lidocaína em diferentes aspectos histopatológicos $(19,20)$. Alguns efeitos adversos quando ela é administrada em altas doses incluem a metahemoglobinemia e hipóxia $(21,22,23)$. Sua toxicidade, ao se combinar com vasoconstritores, tem sido debatida (24, $25,26)$.
A metabolização de xenobióticos, como a Lidocaína, envolve mecanismos enzimáticos complexos. Em algumas situações específicas estas drogas podem provocar lesões em nível hepático ou renal, nestes casos, a análise de parâmetros bioquímicos permite estabelecer a lesão, bem como a sua gravidade. A atividade de aminotransferases, AST (TGO) e ALT (TGP) são largamente usadas na prática clínica como índice sensível, porém não específico de lesão aguda do hepatócito (27-30). Outro parâmetro utilizado na avaliação de possíveis lesões no fígado é a dosagem da bilimubina, a qual é retirada do sangue, conjugada e secretada na bile pelos hepatócitos. Lesão hepática leva ao aumento deste composto no sangue (icterícia), dependendo da sua concentração plasmática pode provocar disfunções fisiológicas $(28,31,32,33)$.

Outra avaliação é feita sobre os níveis de creatinina na circulação, que são relativamente constantes, mas se estiverem elevados, associa-se este fato à disfunção renal, especialmente à disfunção glomerular $(34,35)$.

\section{Material e Métodos}

Para este estudo utilizou-se 40 camundongos machos albinos, de aproximadamente $65 \mathrm{~g}$ cada, Mus musculus brevirostris da linhagem Swiss, fornecidos pelo Biotério Central da PUCPR. Foram separados em caixas com cinco animais e mantidos sob umidade, temperatura e luminosidade naturais com acesso à água potável e ração ad libitum (31). A $\mathrm{DL}_{50}$ calculada foi de $100 \mu \mathrm{g}$ de Lidocaína/g de animal. Durante 14 dias foram realizadas aplicações diárias, intraperitoneal, no mesmo horário, com diferentes doses de Lidocaína $2 \%$ (Astra ${ }^{\circledR}$ ), o grupo 1 recebeu $1 \mu \mathrm{g} / \mathrm{g}$, o grupo 2 recebeu $2,5 \mu \mathrm{g} / \mathrm{g}$ e o grupo 3 recebeu $5 \mu \mathrm{g} / \mathrm{g}$ da droga; o grupo controle (0) recebeu $5 \mu \mathrm{g} / \mathrm{g}$ de solução salina. Concluída esta etapa, os animais foram mortos (tiopental $30 \mu \mathrm{g} / \mathrm{g}$ ) e o sangue coletado por punção cardíaca foi transferido para eppendorfs. Na seqüência as amostras foram centrifugadas a $3500 \mathrm{rpm}$ por 5 minutos, após o sobrenadante (soro) foi coletado e transferido para eppendorfs, que foram congelados e encaminhados para análise. Esta fase foi feita no laboratório PROLAB de Curitiba que utilizou um autoanalisador, Hitachi BM 704, multicanal randômico automatizado. Foram feitos os exames de 
bilirrubina, transaminase pirúvica (TGP) e transaminase oxalacética (TGO) a fim de medir função hepática; e creatinina para medir função renal. Para efeito de comparação utilizaram-se os parâmetros clínicos de normalidade bioquímica para camundongos, nas tabelas de Birchard e Sherding (36). A análise estatística foi feita considerando-se a média \pm desvio padrão, juntamente com o erro padrão de cada grupo. No programa STATISTICA 5.11 a diferença entre as médias foi calculada por análise de variância (ANOVA) com o teste Tukey Acoplado (37). Este protocolo experimental foi avaliado e aprovado pelo Comitê Institucional de Ética Experimental da Pontifícia Universidade Católica do Paraná.

\section{Resulta dos e Discussão}

Segundo Birchard e Sherding (36), os valores normais para cada camundongo da TGP, TGO e Bilirrubina são respectivamente 26 a 77 UI/L, 54 a $269 \mathrm{UI} / \mathrm{L}$ e 0,1 a 0,9 mg/DL De acordo com as observações (gráficos 1, 2 e 3), os resultados obtidos não foram estaticamente significativos e diferentes, quando comparados ao controle. Estudos anteriores, sobre a Lidocaína, mostraram lesões histopatológicas significativas em fígado, rim e sistema nervoso quando o anestésico foi administrado em doses e prazos variáveis, 20 a 30 dias $(9,19$, 20). De acordo com esses estudos, esperavam-se encontrar diferenças nos valores dos marcadores plasmáticos, apontando para as prováveis lesões histológicas; mas os dados obtidos aqui não foram ao encontro desses estudos, é provável que o tempo de exposição ao anestésico (14 dias) tenha sido curto para provocar lesões como descrito. Parece que o tempo de tratamento com o anestésico foi preponderante para obtenção de tais resultados. Outro ponto a ser ressaltado nos dados obtidos foi a diferença encontrada na dose de $1 \mu \mathrm{g} / \mathrm{g}$ na TGO. Em que pese o desvio padrão, o valor da média não foi diferente estatisticamente do controle preconizado. Já no gráfico 4 observa-se o resultado da Lidocaína versus creatinina. Sobre os valores normais que variam entre $0,3 \mathrm{a} 1 \mathrm{mg} / \mathrm{dL}$ (36), verifica-se que não houve alteração estatisticamente significativa. Se o fígado não sofreu injúrias é provável que seu mecanismo enzimático metabolizou a droga e este fato deve ter evitado ações lesivas em nível renal.

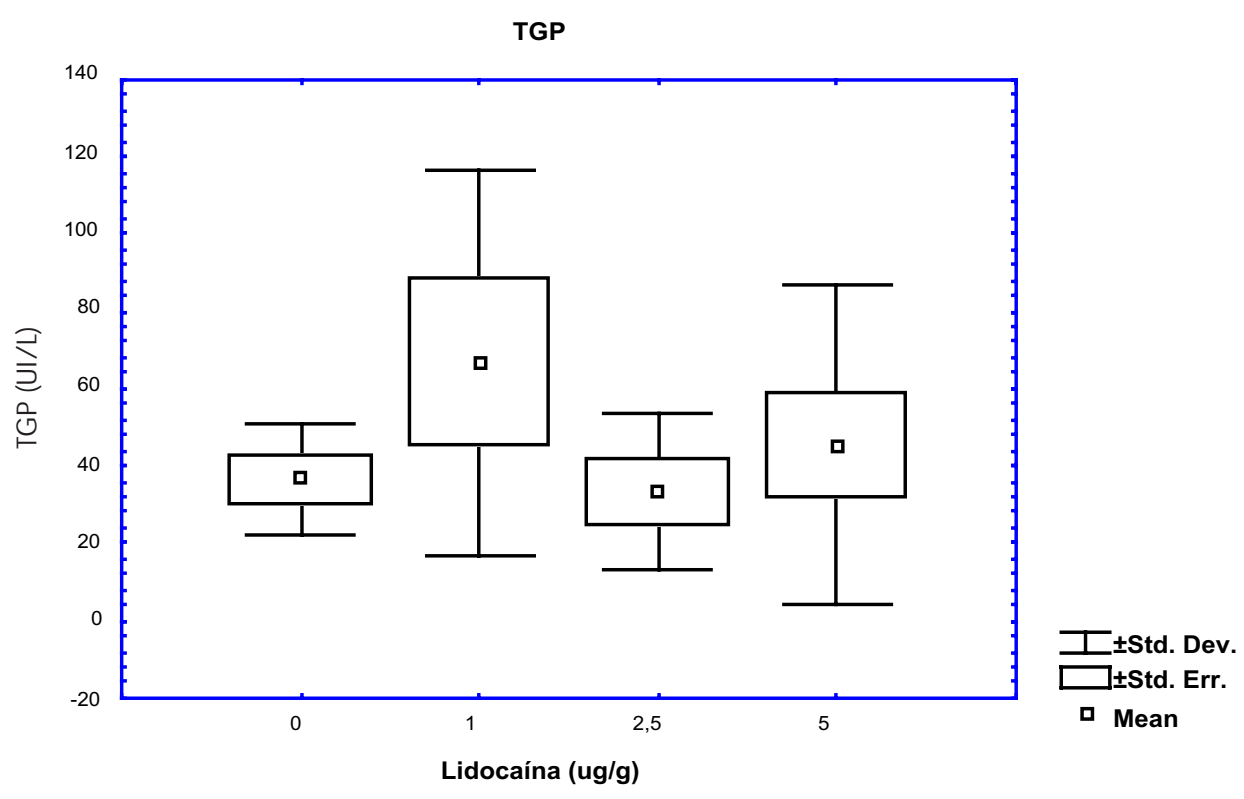

\section{Gráfico 1. Efeito da Lidocaína sobre a TGP. Os resulta dos obtidos não foram significa tiva mente diferentes do controle ( 26 - 77UI/L) (36). Detalhes estatísticos descritos em material e métodos.}




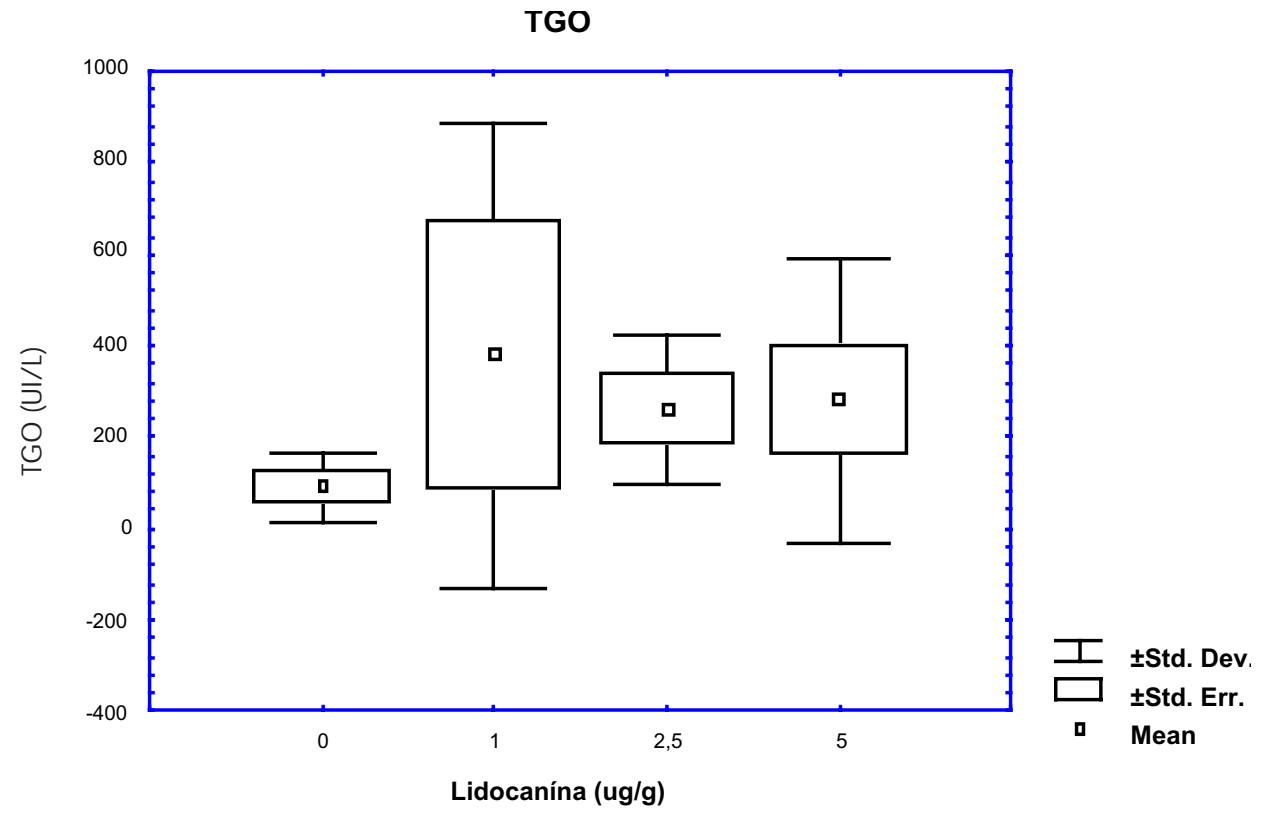

Gráfico 2. Efeito da Lidocaína sobre a TG0. Os resultados obtidos não foram significa tiva mente diferentes do controle ( 54 - 269 UI/L) (36). Detalhes esta tísticos descritos em material e métodos.

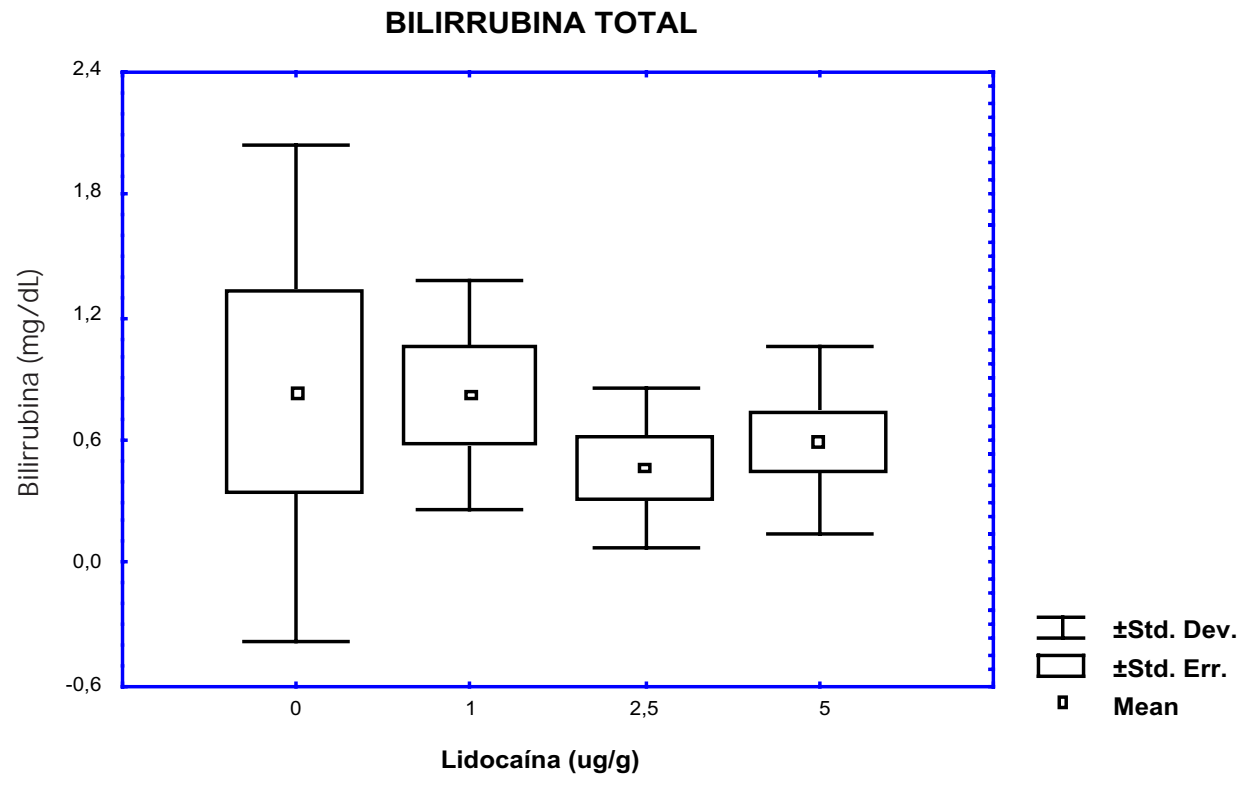

Gráfico 3. Efeito da Lidocaína sobre a Bilirrubina. Os resultados obtidos não foram significa tiva mente diferentes do controle $(0,1-0,9 \mathrm{mg} / \mathrm{dL})(36)$. Detalhes esta tísticos descritos em material e métodos. 


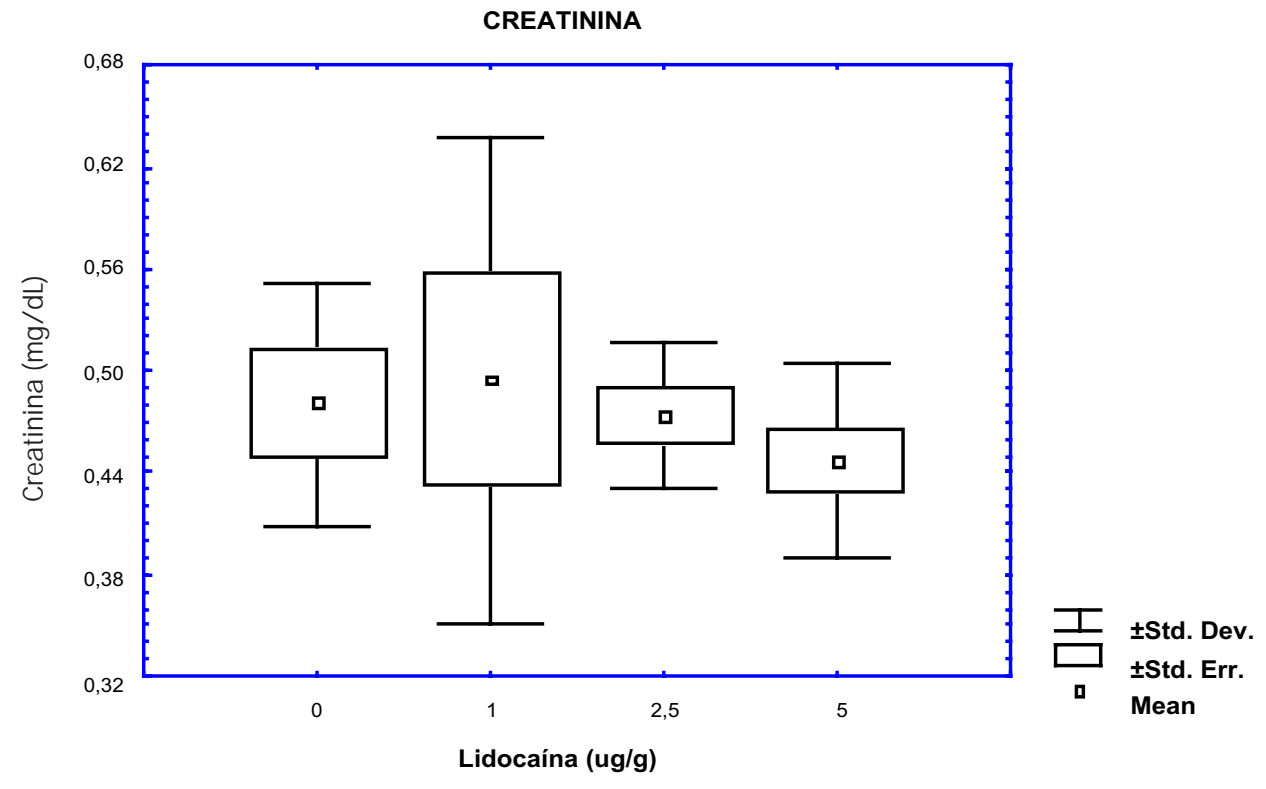

\section{Gráfico 4. Efeito da Lidocaína sobre a Crea tinina. Os resulta dos obtidos não foram significa tiva mente diferentes do controle $(0,3-1,0 \mathrm{mg} / \mathrm{DL})(36)$. Detalhes esta tísticos descritos em material e métodos.}

\section{Conclusões}

Apesar de a literatura apontar para efeitos colaterais em fígado e rim, sob a óptica da histopatologia, os testes, nas condições experimentais assumidas, não mostraram efeitos do anestésico sobre marcadores plasmáticos de lesões hepáticas e renais. Os resultados, quando comparados aos padrões (36), não indicaram mudanças estatisticamente significativas tanto para fígado quanto para rim. É provável que 0 tempo de exposição à droga tenha sido fator preponderante na obtenção de tais resultados. Concluiu-se que o xenobiótico no período de tempo avaliado, 14 dias, não interfere nos níveis normais de TGP, TGO, bilirrubina e creatinina aqui analisados, parecendo que a Lidocaína foi biotransformada e eliminada sem provocar aparente lesão no fígado e/ou nos rins dos camundongos.

\section{Agradecimentos}

Ao técnico Maurício José Barbosa cujo apoio foi fundamental na confecção desta pesquisa.

\section{Referências}

1. Jeske AH. Xylocaine: 50 years of clinical service to dentistry. Tex Dent J 1998; 115(5): 9-13.

2. Melo LL, Sydney RB, Sydney GB. Articaine: a new local anesthetic in dentistry. JBE 2000; $1(2): 80-84$.

3. Covino BG. Pharmacodynamic and pharmacokinetic aspects of local anesthetics. Ann. Chir Gynaecol 1984; 73(3):118-122.

4. Achar S, Kundu S. Principles of office anesthesia: part I. Infiltrative anesthesia. Am Fam Physician 2002; 66(1):91-94.

5. Gammaitoni AR, Alvarez NA, Galer BS. Pharmacokinetics and safety of continuously applied lidocaine patches 5\%. Am J Health Syst Pharm 2002; 15(59):2215-2220.

6. Saranteas T, Tesseromatis C, Potamianou A, Mourouzis C, Varonos D. Stress: induced lidocaine modificatiom in serum and tissues. Eur J Droug Metab Pharmacokinet 2002; 27(4):229-232.

7. Niesel HC, Kaiser H. Dose limits for local anesthetics. Recommendations based on 
toxicologic and pharmacokinetics data. Reg Anaesth 1991; 14(5):79-82.

8. Batista OS, Sant'anna Filho M. Soluções Anestésicas locais disponíveis no mercado brasileiro: doses máximas. Rev. Ass. Paul. Cirur Dent (São Paulo) 2000; 54(3):196-201.

9. Lee HT, Krichevsky IE, Xu H, Ota-Setlik A, D’agati VD, Emala CW. Local anesthetics worsen renal function after ischemia-reperfusion injury in rats. Am J Physiol Renal Physiol 2004; 286(1):111-119.

10. Akerman B, Aström A, Ross S, Telc A. Studies on the absorption, distribution and metabolism of labelled prilocaine and lidocaine in some animals. Acta Pharmacol Toxicol 1966; 24(4):38-45.

11. Mandel WJ, Laks MM, Arieff, AI, Obayashi K, Hayakawa H, Mccullen A. Cardiorenal effects of lidocaine and procaine amide in the conscious dog. Am J Physiol 1975; 228(5):1440-1445.

12. Levy SM, Baker KA. Considerations in differential diagnosis of adverse reactions to local anesthetic: report of case. J Am Dent Assoc 1986; 113(2): 271-273.

13. Elin RJ, Fried MW, Sampson M, RuddeL M, Kleiner DE, Dibisceglie AM. Assessment of monoethylglycinexylidide as measure of liver function for patients with chronic viral hepatitis. Clin Chem 1997; 43(10):1952-1957.

14. Lustig JP, Zusman SP. Immediate complications of local anesthetic administered to 1, 007 consecutive patients. J Am Dent Assoc 1999; 130(4):496-499.

15. Drayer DE, Lorenzo B, Werns S, Reidenberg, MM. Plasma levels, protein binding, and elimination data of lidocaine and active metabolites in cardiac patients of various ages. Clin Pharmacol Ther 1983; 34(1):14-22.

16. Faria FAC, Marzola C. Pharmacology of the Local Anesthetic: general considerations: I Part. JBE 2001; 1(4):82-87.

17. Kalin JR, Brissie RM. A case of homicide by lethal injection with lidocaine. J Forensic Sci 2002; 47(5):1135-1138.
18. Mert T, Gunes Y, Guven M, Gunay I, Gocmen C. Differential effects of lidocaine and tramadol on modified nerve impulse by 4-aminopyridine in rats. Pharmacology 2003; 69(2):68-73.

19. Kishimoto T, Bollen AW, Drasner K. Comparative spinal neuroxicity of prilocaine and lidocaine. Anesthesiology 2002; 97(5):12501253.

20. Tomazeli L. Análise histopatológica da toxicidade de prilocaína 3\% com felipressina (citanest 3\% com octapressin) sobre camundongos. Cunitiba; 2001 [Monografia] - Pontifícia Universidade Católica do Paraná.

21. Mets B, Hickman R, Allin R, Van Dyk J, Lotz Z. Effect of hypoxia on the hepatic metabolism of lidocaine in the isolated perfused pig liver. Hepatology 1993; 17(4):668-676.

22. Essink-Tebbes CM, Wuis EW, Liem KD, Van Dongen, RT, Hekster YA. Safety of lidocaineprilocaine cream application four times a day in premature neonates: a pilot study Eur J Pediatr 1999; 158(5):421-423.

23. Touma S, Jackson JB. Lidocaine and prilocaine toxicity in a patient receiving treatment for mollusca contagiosa. J Am Acad Dermatol 2001; 44(2):399-400.

24. Volpato MC, Ranali J, Amaral IM, Demetrio CG, Chalita LV. Acute toxicity (LD50 and CD50) of lidocaine and prilocaine in combination with adrenaline and felypressin. Indian J Dent Res 1999; 10(4):138-144.

25. Wahl MJ, Overton D, Howell J, Siegel E, Schmitt MM. Muldoon M. Pain on injection of prilocaine plain vs. lidocaine with epinephrine. A prospective double-blind study. J Am Dent Assoc 2001; 132(10):1396-1401.

26. Baluga JC, Casamayou R, Carozzi E, López N, Anale R, Borges R, et al. Allergy to local anaesthetics in dentistry. Myth or reality?Allergol Immunopathol 2002; 30(1):14-19.

27. Rizzi V, Cioshi B, Cartei G, Bertolissi A, Tabaro G, Marsilio P. Liver function test and lidocaine metabolism during i.v. CMF therapy in breast cancer. Anticancer drugs 1996; 7(8):865-850. 
28. Devlin TM. Manual de Bioquímica com correlações clínicas. São Paulo: Edgard Blucher; 1998.

29. Giannini E, Botta F, Fasoli A, Ceppa P, Risso D, Lantieri PB, et al. Progressive liver functional impairment is associated with an increase in ST/ALT ratio. Dig Dis Sci 1999; 44(6):12491253.

30. Gehringer MM, Shephard ED, Downing TG, Wiegand C, Neilan BA. An investigation into the detoxification of microcystin-LR by the glutathione pathway in Balb/c mice. Int J Biochem Cell Biol 2004; 36(5):931-941.

31. Brito AS. Manual de Ensaios Toxicológicos in Vivo. São Paulo: Universidade Estadual de Campinas UNICAMP; 1994.

32. Shiffman ML, Luketic V A, Sanyal AJ, Duckworth PF, Purdum PP, Contos MJ, et al. Hepatic lidocaine metabolism and liver histology in patients with chronic hepatitis and cirrhosis. Hepatology 1994; 19(4):933-940.
33. CLayton TA, Lindon JC, Everett JR, Charuel C, Hanton G, Le Net JL, et al. Hepatotoxin-induced hypercreatinaemia and hypercreatinuria: their relationship to one another, to liver damage and to weakened nutritional status. Arch Toxicol 2004; 78(2):86-96.

34. Nishiyama T, Yokoyama T, Hanaoka K. Liver and renal function after repeated sevoflurane or isoflurane anesthesia. Can J Anaesth 1998; 45(8):789-793.

35. González GA, Illera JC, Silván G, Lorenzo PL, Illera $\mathrm{M}$. Changes in hepatic and renal enzyme concentrations and respiratory rates in new zeland white rabbits after anesthetic treatments. Contemp Top Lab Anim Sci 2002; 41(6):2.

36. Birchard SJ, Sherding RG. Manual Saunders clínica de pequenos animais. São Paulo: Roca; 1998.

37. Magnusson W, Mourão G. Estatística sem matemática: a ligação entre as questões e a análise. Londrina: Planta; 2003.

Recebido em / Received: June 21, 2005.

Revisado em / Revised: July 27, 2005. Aceito em / Accepted: December 2, 2005. 